ISBN 978-981-14-1684-2

Proceedings of 2019 the 9th International Workshop on Computer Science and Engineering

(WCSE 2019 SUMMER)

Hong Kong, 15-17 June, 2019, pp. 748-752

doi: $10.18178 /$ wcse. 2019.06 .111

\title{
Usability Tests of Thai Mobile Banking UI Design
}

\author{
Inthraporn Aranyanak ${ }^{+}$ \\ Department of Computer Science \\ King Mongkut's Institute of Technology Ladkrabang \\ Bangkok, Thailand
}

\begin{abstract}
Nowadays, many banking customers process their transactions via mobile banking applications because of the convenience. This study examines five well-known Thai mobile banking applications to measure their usability and compares their UI design from the perspective of nine main functions. An analytical tool for prototypes and the System Usability Scale (SUS) were used to collect and analyse data. The usability test of the five mobile banking applications in this paper involves three usability metrics: effectiveness showing the completion rate, efficiency showing the time used to successfully complete a task, and satisfaction using SUS to evaluate users' satisfaction with the product. This paper presents problems found in poor UI design and suggests how to improve mobile banking UI design.
\end{abstract}

Keywords: Human and Computer Interface, Usability Testing, Mobile Banking Application, User Experience, User Interface Design

\section{Introduction}

According to the Bank of Thailand, the use of mobile banking and internet banking in 2018 showed that the number of customer applications for the mobile banking service was nearly 38 million compared to just over 23 million for internet banking [1]. Therefore, mobile banking applications will become more popular for customers to check their bank account anywhere and at any time. Financial transactions on a mobile application can be done faster than going to a bank branch and are free of charge. For those with a mobile phone and a bank account, $67 \%$ of mobile banking users are aged 18 to 29 and $58 \%$ of are aged 30 to 44 . By comparison, only $18 \%$ of customers older than 60 have used mobile banking applications [2]. One of the commonest reasons why people do not make a financial transaction via mobile banking is a concern about the security of mobile payments, especially involving a large amount of money. This might be because they are afraid if something goes wrong they cannot talk to bank staff directly. Another reason is some people are not good at using technology, so they are afraid of making mistakes and think that it is too hard for them to use. Therefore, security, trust, and ease of use should be the main factors for user interface designers to incorporate into the design of a mobile banking application. The bad design of the elements in the interface can make people less trusting, such as, grammatical errors, broken links, inconsistency, confusing icons, etc. Therefore, conducting research on UI and UX mobile banking design is necessary to provide effective guidelines.

The purpose of this study is to observe the satisfaction level of Thai mobile banking customers and conduct a usability test comparing five well-known Thai mobile banking applications. The results present a satisfaction survey of user interface design and an analysis of good and bad interface design among those mobile banking applications. The usability tests provide generalisable information that can be applied to other mobile banking designs to enhance users' experience.

+ Corresponding author. Tel.: + 662-329-8400; fax: +662-329-8412.

E-mail address: Inthraporn.ar@kmitl.ac.th. 


\section{Background and Related work}

\subsection{UX and UI for mobile application design}

A mobile application, also called a mobile app, is a software application operating on a mobile device. To develop a mobile app, you must know the platform on which it is to be deployed. Native mobile applications for iOS and Android have different operating system-specific features; they also differ in terms of structure and flow, such as, navigation patterns, layout, typography, etc. You need to consider these design differences to provide the best user experience. The biggest challenge in designing a mobile app interface is the limitation of screen size. This leads to many UI design constraints. For instance, information or actions on a screen should not be too complicated. Types of typography can affect legibility; some studies found that sans serif is easier to read at very small sizes while serifs has more visual noise and detract from the main body shape of the letter form [6]. Therefore, common typefaces used on a mobile app have similar characteristics as sans serif. Google Android uses Roboto and Noto, Apple uses the San Francisco family of typefaces as the standard typefaces for mobile UI design. For a legible font size, text should be at least 11 points, so users can read it at a typical viewing distance without zooming. The size of touch targets should be between $7-10 \mathrm{~mm}$, which is equivalent to the smallest average fingertip.

Designing software based on user experience becomes more important these days if you want your product to stand apart from competitors. It is important because it tries to fulfil the user's needs and provide a strong value proposition that can result in user growth and continued use. Beyond that, it makes for lower maintenance costs. A well-known technique used to measure a product by testing it on users is a usability test.

Most Thai mobile banking systems have used surveys to determine behaviour and satisfaction with using mobile banking service. They found that most Thai banking customers do not use a mobile banking service because of security concerns, poor UI design, and lack of usability [3,4,5]. There has not yet been a study to investigate the usability of UI design that compares Thai mobile banking apps. Hence, this paper describes usability tests of five mobile banking applications and illustrates good and bad UI and UX design, so that application designers can have useful guidelines for better customer experience.

\subsection{Usability testing}

Developing software based on User-Centered Design (UCD) comprises a variety of methods, techniques, and practices, each applied at different points during the product development process. There are many ways to collect qualitative and quantitative data from users, such as, surveys, interviews, focus groups, prototyping, usability testing, etc. Usability testing is a technique to collect empirical data while observing representative end users using the product to perform actual tasks. The results of the usability test will provide sufficient evidence to help designers and developers improve the usability of their products. There are several usability models and attributes to evaluate the usability of the product [7]. For instances, the Norman model is aimed at suggesting design principles, which can make the product less complex and easier to use. The Nielsen model defined five principles to assess how easy user interfaces are to use: learnability, efficiency, memorability, errors, satisfaction. The ISO/IEC 9126-4 recommended that usability metrics should include these 3 elements:

- effectiveness, which is the ability to perform a task successfully. It can be calculated by measuring the completion rate and number of errors made performing specific tasks.

- efficiency, which refers to the amount of effort users need to achieve their goals. It is mostly measured by the time used to successfully complete a task.

- satisfaction, which is a measure of how users feel about the product. Rating scale questions are commonly used to evaluate users' satisfaction with the product.

\section{Methods}

\subsection{Procedure}

Five popular mobile banking app with different UI design and nine common functions were selected from the result of an online survey including general questions, such as, their current mobile banking app, 
type of logging in, security features, ease of use and convenience, functions used on mobile a banking app, etc. An example of the main screens of each selected mobile banking app are presented in Fig 1.

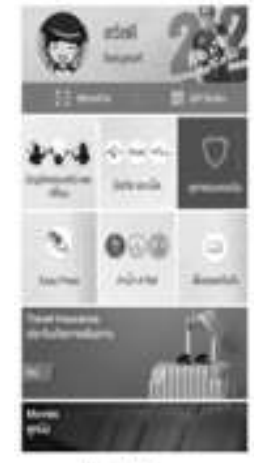

Bank 1

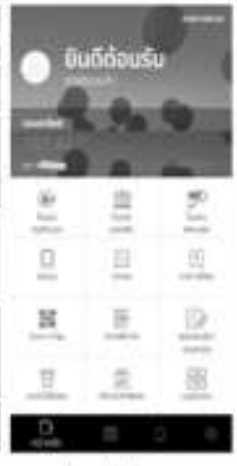

Bank 2

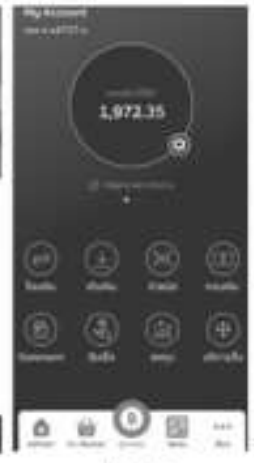

Bank 3

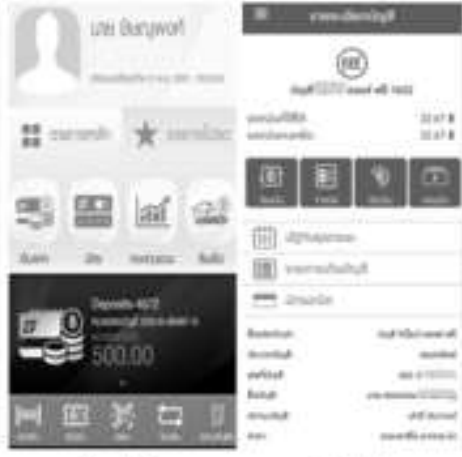

Bank 4

Bank 5

Fig. 1: An example of the main screen of the five selected mobile banking app.

Nine most common functions are "Transferring money to other banks" (F1), "Adding to favorite" (F2), "Paying through QR code" (F3), "Checking balance" (F4), "Transferring money to the same bank" (F5), "Paying through Bar code" (F6), "Privilege" (F7), "Transferring money through PromptPay" (F8), "Checking transaction" (F9). Participants had two trials to practice the process of doing the usability test before an actual test run. Each participant was asked to read and sign a consent form before the test. They were asked to complete nine tasks on each of five mobile banking apps. After each application, a System Usability Scale (SUS) survey was used to measure the participants' perceptions of usability. The study finished within 45 minutes.

\subsection{Participants and tools}

Maze, an analytical tool for prototypes, and System Usability Scale (SUS) were used to measure the usability of the mobile banking app prototypes. Thirty mobile banking customers participated in the study: 22 males and eight females ranging in age from 18 to 24 for 26 participants and between 35-55 for four participants. Maze was used to define missions, collect actionable insights and analyse the usability test. SUS comprising 10 questions was also used to provide assessments of usability.

\section{Results}

\subsection{MAZE - Analytics for prototypes}

This paper shows the overall data provided by MAZE. It includes the percentage of task success, average duration of each task success, and click heatmaps.

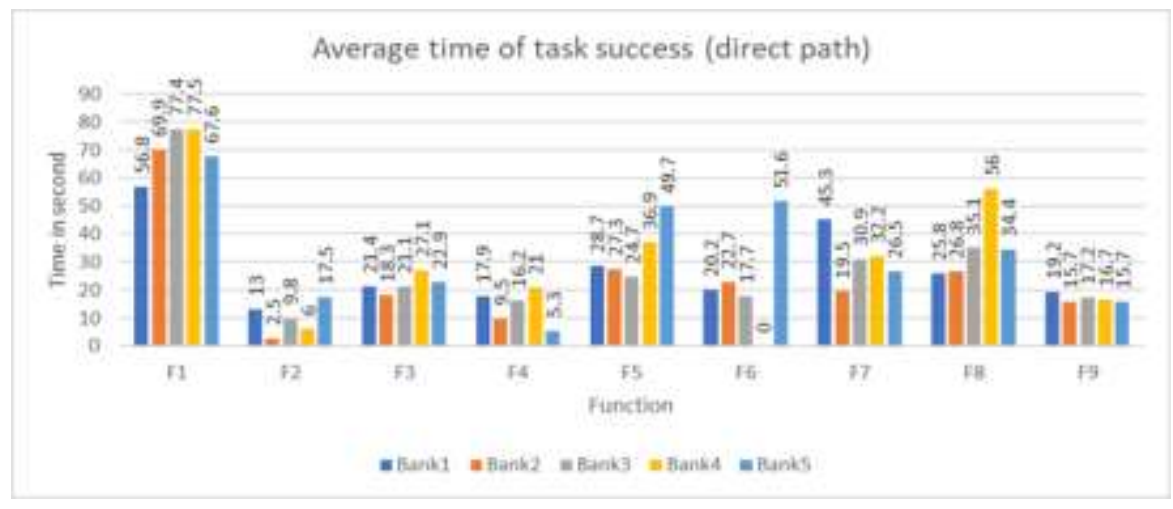

Fig. 2: Average time of task success.

Fig. 2 shows the average time to success for each task in seconds. The total average times (excluding F6, since Bank4 doesn't have this function) are 228.1, 189.5, 232.4, 273.4, 239.6, respectively. The usability test via MAZE demonstrates that there are many UI design factors affecting the effectiveness and efficiency 
metrics. For instance, ambiguous words used for F2 meaning "Adding to favorite", in Bank5 that uses a different word "Adding payee" where users might get confused with the meaning. As shown in Fig.3, users misclicked the button.

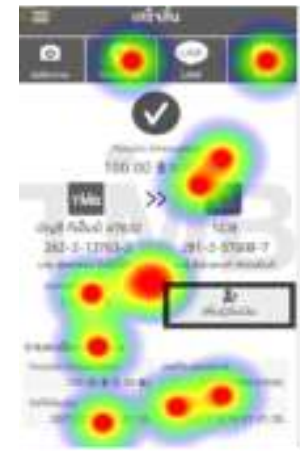

Fig. 3: "Adding to favorite".

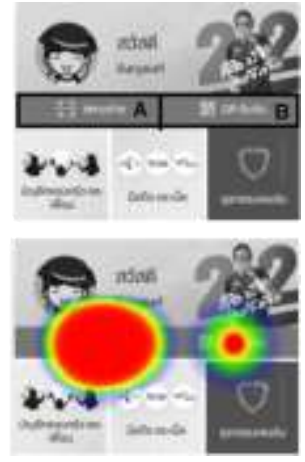

Fig. 4: Icons with multiple meaning.

\section{ICON A}

\section{gig ICONB}

Fig.5: Icon A and B

Fig. 4 shows the main UI screen of Bank1. When participants were asked to complete "Paying through QR code" (F3) and "Paying through Bar code" (F6) they were confused between the icon A for scanning to pay and the icon B for generating a QR code for payment as shown in Fig. 5. In this case, the icon A is the action for F3 and F6, but the icon B is located nearby, which can also be interpreted for F3 and F6. Hence, some participants mistakenly clicked on the icon B.

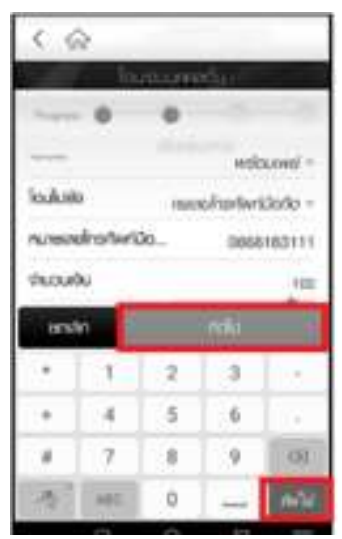

Fig. 6: "Next" button.

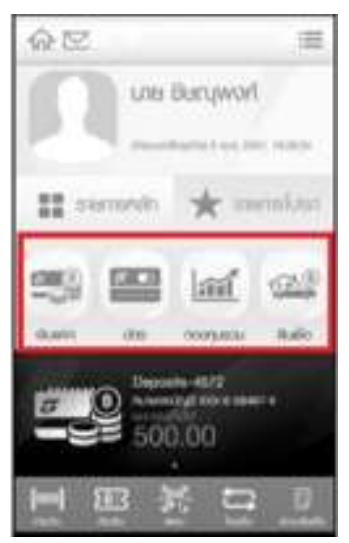

Fig. 7: Hidden function.

The "Next" button in Fig 6 shows the same label for the next action with the keyboard, which causes confusion. Moreover, the function of "Privilege" shown in Fig.7 is in the hidden tab bar which does not look like it can be slid to the right which makes it difficult for participants to find it.

\subsection{System usability scale (SUS) with adding an adjective rating Scale}

This study used SUS containing 10 questions with a seven-point Likert scale. The mean SUS score ratings corresponding to the seven adjective ratings are as follows: 12.5 is "Worst Imaginable", 20.3 is "Awful", 35.7 is "Poor", 50.9 is "OK", 71.4 is "Good", 85.5 is "Excellent", 90.9 is "Best Imaginable" [7]. The results of this study from SUS are shown in Table2. 
Table 1. The mean of each SUS question with the seven adjective ratings.

\begin{tabular}{|c|l|l|l|l|l|l|l|l|l|l|c|}
\hline Bank & Q1 & Q2 & Q3 & Q4 & Q5 & Q6 & Q7 & Q8 & Q9 & Q10 & Average SUS Score \\
\hline 1 & 5.43 & 2.77 & 4.93 & 2.70 & 5.47 & 2.17 & 5.03 & 2.53 & 5.17 & 2.87 & 71.7 \\
2 & 5.07 & 2.73 & 5.20 & 2.33 & 5.23 & 2.33 & 5.03 & 2.47 & 4.97 & 2.80 & 71.4 \\
3 & 5.57 & 2.70 & 5.43 & 2.83 & 5.73 & 2.50 & 5.17 & 2.53 & 5.30 & 2.93 & 72.8 \\
4 & 2.93 & 4.60 & 2.97 & 4.50 & 4.07 & 4.30 & 2.87 & 4.77 & 6.00 & 4.33 & 43.9 \\
5 & 2.40 & 5.50 & 2.77 & 4.63 & 4.03 & 4.80 & 2.57 & 5.73 & 5.27 & 4.80 & 35.9 \\
\hline
\end{tabular}

The perceived learnability of the mobile banking application can also be measured by questions 4 and 10 from the SUS ("I think I would need the support of a technical person to be able to use this system" and "I needed to learn a lot of things before I could get going with this system"). Table 1 shows that the rating scores of Bank1, Bank2, and Bank3 for question 4 and 10 are less than the level of 3 compared to Bank4 and Bank5 that got higher rating scores. The findings imply that the UI designs of Bank4 and Bank5 are too complicated for users to learn easily by themselves. Based on several studies, an average SUS score above 68 would be considered acceptable and any application below needs improvement in its UX/UI design. The average SUS Scores of Bank 1, Bank2, and Bank3 are in the "Good" level, however, the average SUS Scores of Bank 4 and Bank5 are in the "Poor" level.

\section{Conclusion}

The online usability testing tool, MAZE, can help UX designers observe and analyse user behaviour by showing click locations, paths, and time spent on each screen. UX and UI designers can use this information to identify which parts of the UI design need to be improved. The results from MAZE correlate well with those of SUS: the average times to task success for Bank 4 and Bank 5, for example, are longer than for other banks, correlating with an average "poor" SUS score for the same banks. Moreover, the evaluation of learnability for Bank 4 and Bank 5 also indicates that users feel they need technical support to use the application effectively. The usability tests demonstrated that users were confused by misleading words and ambiguous icons, especially when they are located close to each other, often causing erroneous clicks. Moreover, hiding frequently used functions in a hamburger icon slowed users' ability to find the associated functions. Using card sorting techniques and prototype testing can help UX and UI designers to better organise the information architecture of their systems and prevent some of the problems identified here.

In addition, this study found that the tasks were completed quickly since the user interfaces were designed to have shorter paths. The most frequently used functions should be found easily on the main screen. Designing UI with multiple paths also helps users to accomplish their task efficiently. Using ambiguous words as labels, icons with mutliple interpretations, and bad navigation design can all cause users to become disoriented and affect user retention rates.

\section{References}

[1] Bank of Thailand, http://www2.bot.or.th/statistics/BOTWEBSTAT.aspx?reportID=688\&language=ENG, last accessed 2018/11/28.

[2] Consumers and Mobile Financial Services 2016, www.federalreserve.gov/pubs/orderform.pdf. , last accessed 2019/1/16.

[3] Propon Hanharirak. Security of mobile banking applications. Masters thesis. King Mongkut's University of Technology North Bangkok, 2016.

[4] Nattha Hatthapan. The satisfactions of users in KTB netbank and $K$-cyber banking/ $K$ - mobile banking plus in Bangkok. Maters thesis. Rajamangala University Of Technology Rattanakosin, 2016.

[5] Kanjana Jantatueng. Behaviors of customers' K-Mobile Banking Plus services organized by Kasikorn Thai Banks in Chiang Saen district, Chiangrai province. Master thesis. Chiang Rai Rajabhat University, 2018.

[6] Hoober, Steven. Mobile Input Methods. UXmatters, November 1, 2012. Retrieved January 18, 2013.

[7] Aaron Bangor, Philip Kortum, James Miller. Determining What Individual SUS Scores Mean: Adding an Adjective Rating Scale. Journal of Usability Studies Vol. 4. Issue 3, May, 2009. 